Review

\title{
Neutrinos in dense quark matter and cooling of compact stars
}

\author{
D. Blaschke ${ }^{\mathrm{a}, \mathrm{b}, *}$, J. Berdermann $^{\mathrm{c}}$ \\ a Institute for Theoretical Physics, University of Wroclaw, Poland \\ ${ }^{\mathrm{b}}$ Bogoliubov Laboratory for Theoretical Physics, JINR Dubna, Russia \\ ${ }^{c}$ DESY, Platanenallee 6, D-15738 Zeuthen, Germany
}

\section{A R T I C L E I N F O}

\section{Keywords:}

Neutron star

Color superconductivity

Neutrino emissivity

Cooling processes

Quark matter

\section{A B S T R A C T}

We discuss that observational constraints on neutrino cooling processes may restrict the spectrum of quark matter phases admissible for compact star interiors.

(C) 2010 Elsevier B.V. All rights reserved.

\section{Introduction}

The existence of quark matter in compact star (CS) interiors has been debated controversially since observations of the thermal emission from objects like RXJ 1856.5-3754 [1] indicate large masses and/or luminosity radii requiring a stiff equation of state (EoS) at high densities. Although this seemingly excludes a phase transition accompanied with a softening of the EoS, it has been demonstrated [2] that modern QCD motivated theories could fulfill such observational constraints, e.g., due to the presence of vector mean fields which stiffen the quark matter EoS [3]. Investigations of color superconducting quark matter phases (for a recent review, see [4]) show that large diquark pairing gaps of $\sim 10-100 \mathrm{MeV}$ are possible, which lower the critical density for the chiral symmetry restoration due to their competition with the chiral condensate. This entails early quark deconfinement unless color forces remain strong enough to confine even (almost) massless quarks in a new phase of chirally symmetric hadronic matter, the hypothetic quarkyonic phase [5,6]. Here, we will not yet consider quarkyonic matter, but discuss the viability of three-flavor (CFL), two-flavor (2SC) and one-flavor (dCSL) color superconducting phases under constraints for mass and radius as well as CS cooling.

Spin-0 phases which pair quarks of different flavor and opposite spins with large gaps (2SC and CFL) might be too fragile to withstand the stress of flavor asymmetry and strong magnetic fields in neutron stars. It is not clear yet whether the occurrence of the celebrated CFL phase in the core of a hybrid star leads necessarily to a gravitational instability [3] as a result of the strong softening of the EoS (for an exception, see [7]). Disregarding the hadronic shell even interesting mass twin sequences are possible [8]. If hybrid stars with CFL quark matter cores can form a third CS family, this might allow for a new paradigm to explain the Janus-faced CS phenomenology: large luminosity radii vs. millisecond rotation periods, fast cooling vs. slow cooling etc.

After the new color superconductivity phases with large Spin-0 pairing gaps (2SC and CFL) were suggested in 1997 , a discussion of late time cooling scenarios has been performed in $[9,10]$, followed by a full transport calculation with a nontrivial temperature profile evolution in hybrid stars with CSC quark matter cores [11]. These calculations showed that the pairing pattern where all quarks are paired with large gaps delay the cooling and may be excluded by the observation of fast coolers like the Vela pulsar, unless they form a third family.

In these works as well as in the detailed study in Ref. [12] the fact was ignored that in the 2SC phase the quarks with one of the colors (e.g., the blue ones) remain unpaired so that the fast direct Urca (DU) process is operative, entailing too fast

\footnotetext{
* Corresponding author at: Institute for Theoretical Physics, University of Wroclaw, Poland.

E-mail address: blaschke@ift.uni.wroc.pl (D. Blaschke).
} 
cooling. For heuristic purposes, a residual single-color pairing of the blue quarks (X-gap) has been introduced [13] and it has been found that the best cooling phenomenology would be obtained for hybrid stars with superconducting quark matter cores in the $2 \mathrm{SC}+\mathrm{X}$ phase when this smallest gap is in the range between $100 \mathrm{keV}$ and $1 \mathrm{MeV}$, with a decreasing density dependence $[14,15]$. An alternative to the postulated $2 S C+X$ phase which provides a microscopic approach to the pairing pattern in accordance with cooling phenomenology is the isotropic color-spin-locking (CSL) phase [16] for which quarks of the same flavor are paired in a spin- 1 state. This phase is thus rather inert against the neutron star stress.

Recently, it has been argued that the different quark flavors could occur sequentially, i.e. at different threshold densities in neutron star matter $[17,18]$. In analogy to the neutron drip in the crust, a down-quark drip density can occur in the core, when d-quarks undergo a chiral restoration and partial nucleon dissociation leads to the formation of a superconducting single-flavor subphase (d-CSL) immersed in nuclear matter. This mixture of d-CSL and nuclear matter can extend from the interior to the crust core boundary in stable hybrid star configurations which fulfill the stringent mass-radius constraints mentioned above. It also bears interesting perspectives for the neutrino transport and cooling properties $[17,19]$ which we discuss now in a bit more detail.

\section{Neutrino emissivity and deep crustal heating}

According to [20], two interesting phenomena, the superbursters and the soft X-ray transients may be probes for the existence of CS deep crustal heating processes subject to the following constraints

1. A thin baryonic crust of the star with a width between 100 to $400 \mathrm{~m}$.

2. An energy release of $1-100 \mathrm{MeV}$ per accreted nucleon by conversion at the crust-core boundary.

3. The thermal conductivity has to be in the range of $10^{19}-10^{22} \mathrm{erg} \mathrm{cm}^{-1} \mathrm{~s}^{-1} \mathrm{~K}^{-1}$.

4. The fast DU neutrino emissivity process should be strongly suppressed or not operational.

Following the suggestion of Refs. [21,22], the conversion of nuclear matter to CFL strange quark matter, powered by continuous accretion in a LMXB may provide a mechanism for the deep crustal heating. As the nuclei penetrate into the quark matter core a conversion energy between 1 to $100 \mathrm{MeV}$ is released per accreted nucleon. This heats the core until an equilibrium temperature is reached and the heating is balanced by neutrino emission. As the DU neutrino emission process is strongly suppressed by large CFL gaps, the heat produced at the crust-core boundary is not radiated away but conducted so that the fusion ignition condition gets fulfilled.

In [17] we have shown that a d-quark CSL/nuclear phase mixture can also fulfill the above constraints on the deep crustal heating mechanism. In particular, we estimated the heat per accreted nucleon from the d-quark drip due to partial chiral restoration to the order of $10 \mathrm{MeV}$ and the DU process which we exclude in the nuclear subphase also does not occur in the d-CSL subphase since the Fermi sea of up quarks is closed. An interesting question for the d-CSL/nuclear matter phase to be clarified is the possible role of confining interactions between the colored d-quarks which would energetically forbid a rather dilute d-quark admixture and sets a threshold for the d-quark drip. It may well be that the proper account for confining interactions within a chiral model theory would lead to a modification of the nucleon properties rather than to their partial dissociation at the chiral restoration for the down quarks. A modified quarkyonic phase for isospin asymmetric matter can be suggested.

\section{Perspectives}

Hybrid Stars with a mixture of nuclear matter and d-CSL quark matter phases fulfill not only stringent constraints for large radii and masses but also provide a viable deep crustal heating process. This mixed phase should probably better described as a kind of quarkyonic matter, once this gets accessible to a theoretical description. Further investigation needs the idea that CFL quark core stars form a third CS family providing a possible explanation of a different class of CS phenomena as, e.g., GRBs [23,24], SGRs, AXPs and XDINs [25].

\section{Acknowledgements}

We are grateful to our colleagues for discussions and collaboration. D.B. acknowledges an EPS Fellowship, and partial support by MNiSW grant No. N N 202 231837, by RFBR grant No. 08-02-01003-a and by CompStar, a Research Networking Programme of the European Science Foundation.

\section{References}

[1] J.E. Trümper, V. Burwitz, F. Haberl, V.E. Zavlin, Nuclear Phys. B Proc. Suppl. 132 (2004) 560.

[2] M. Alford, D. Blaschke, A. Drago, T. Klähn, G. Pagliara, J. Schaffner-Bielich, Nature 445 (2007) E7. arXiv:astro-ph/0606524.

[3] T. Klähn, D. Blaschke, F. Sandin, Ch. Fuchs, A. Faessler, H. Grigorian, G. Röpke, J. Trümper, Phys. Lett. B 654 (2006) 170.

[4] M.G. Alford, A. Schmitt, K. Rajagopal, T. Schäfer, Rev. Modern Phys. 80 (2008) 1455.

[5] L. McLerran, R.D. Pisarski, Nuclear Phys. A 796 (2007) 83.

[6] A. Andronic, et al. arXiv:0911.4806 [hep-ph].

[7] G. Pagliara, J. Schaffner-Bielich, Phys. Rev. D 77 (2008) 063004. 
[8] F. Sandin, D. Blaschke, Phys. Rev. D 75 (2007) 125013.

[9] D. Blaschke, T. Klähn, D.N. Voskresensky, Astrophys. J. 533 (2000) 406.

[10] D. Page, M. Prakash, J.M. Lattimer, A. Steiner, Phys. Rev. Lett. 85 (2000) 2048.

[11] D. Blaschke, H. Grigorian, D.N. Voskresensky, Astron. Astrophys. 368 (2001) 561.

[12] P. Jaikumar, C.D. Roberts, A. Sedrakian, Phys. Rev. C 73 (2006) 042801.

[13] H. Grigorian, D. Blaschke, D. Voskresensky, Phys. Rev. C 71 (2005) 045801.

[14] D. Blaschke, D.N. Voskresensky, H. Grigorian, Nuclear Phys. A 774 (2006) 815.

[15] S. Popov, H. Grigorian, D. Blaschke, Phys. Rev. C 74 (2006) 025803.

[16] D.N. Aguilera, D. Blaschke, M. Buballa, V.L. Yudichev, Phys. Rev. D 72 (2005) 034008.

[17] D. Blaschke, F. Sandin, T. Klähn, J. Berdermann, Phys. Rev. C 80 (2009) 065807. arXiv:0807.0414 [nucl-th]; AIP Conf. Proc. 1038 (2008) 183.

[18] D. Blaschke, F. Sandin, T. Klähn, J. Phys. G 35 (2008) 104077.

[19] D.B. Blaschke, J. Berdermann, AIP Conf. Proc. 964 (2007) 290

[20] M. Stejner, J. Madsen, Astron. Astrophys. 458 (2006) 523.

[21] D. Page, A. Cumming, Astrophys. J. 635 (2005) L157.

[22] A. Cumming, J. Macbeth, J.J.M. in 't Zand, D. Page, Astrophys. J. 646 (2006) 429.

[23] R. Ouyed, R. Rapp, C. Vogt, Astrophys. J. 632 (2005) 1001. arXiv:astro-ph/0503357.

[24] J. Berdermann, et al., Prog. Part. Nucl. Phys. 57 (2006) 334.

[25] B. Niebergal, R. Ouyed, R. Negreiros, F. Weber, arXiv:0906.3043 [astro-ph.HE]. 